\title{
Time resolved X-ray spectroscopy of V404 Cyg during the June 2015 outburst
}

\author{
Celia Sánchez-Fernández* \\ INTEGRAL Science Operations Center, European Space Astronomy Centre (ESAC), Camino \\ Bajo del Castillo s/n, Villafranca del Castillo, 28692 Villanueva de la Cañada, Madrid, Spain \\ E-mail: Celia.Sanchezesciops.esa.int
}

\section{Jari Kajava}

Tuorla Observatory, Department of Physics and Astronomy, University of Turku, Väisäläntie 20, FIN-21500 Piikkiö, Finland

E-mail: jari.kajava@utu.fi

\section{Sara Elisa Motta}

University of Oxford, Department of Physics, Astrophysics, Denys Wilkinson Building, Keble Road, Oxford OX1 3RH, UK

E-mail: sara.mottaephysics.ox.ac.uk

\section{Erik Kuulkers}

INTEGRAL Science Operations Center, European Space \& Technology Centre, Keplerlaan 1, Postbus 299, 2200 AG Noordwijk The Netherlands

E-mail: Erik.Kuulkersesciops.esa.int

\begin{abstract}
Hard X-ray spectra of black hole binaries (BHB) are produced by the inverse Comptonization of soft seed photons by hot electrons near the black hole. The slope of the resulting energy spectra is governed by two main parameters: the electron temperature $\left(T_{\mathrm{e}}\right)$ and the optical depth $(\tau)$ of the emitting plasma. The extremely bright outburst of V404 Cyg in June 2015, provides a unique data set to perform time resolved spectroscopy and study in detail the evolution of the parameters describing the Comptonizing plasma. We present here the results of IBIS/ISGRI spectral analysis in the 20-200 keV energy range over the period 18-28 June 2015.
\end{abstract}

11th INTEGRAL Conference Gamma-Ray Astrophysics in Multi-Wavelength Perspective, 10-14 October 2016

Amsterdam, The Netherlands

${ }^{*}$ Speaker. 


\section{Introduction}

V404 Cyg is a transient Low-Mass X-ray binary (LMXB) consisting of a $9.0_{-0.6}^{+0.2} \mathrm{M}_{\odot}$ black hole (BH) accreting mass from a K3 III companion [1]. The system orbital period is $6.5 \mathrm{~d}$ [2]. It is located at a distance $d=2.39 \pm 0.14 \mathrm{kpc}$ [3]. V404 Cyg was first detected in optical wavelengths during two outbursts in 1938 and 1956 [4] and later in X-rays during a third outburst in 1989 [5, 6]. After $\sim 26$ years in quiescence, the onset of a new outburst was detected by Swift/BAT, MAXI and Fermi/GBM on 15 June 2015 [7, 8, 9]. The outburst lasted until early-August 2015 [10]. During the first ten days of the June 2015 outburst, the source exhibited violent flaring activity on time scales of sub-seconds to hours in all the wavelengths: $\gamma$-rays [11]; X-rays [12, 13, 14, 15]; Optical [16, 17, 18]; Infrared [19]; Millimeter/Sub-millimeter and Radio [20]. In some major flares, V404 Cyg reached fluxes around 50 and $40 \mathrm{Crab}$ in soft and hard X-rays respectively [21, 12]. The peak of the outburst was reached on June 26th, and the flux dropped immediately afterwards [22, 23] slowly fading to quiescence over the subsequent weeks [10].

V404 Cyg was observed by INTEGRAL in a series of Target of Opportunity observations scheduled between 17 June, 2015 and 13 July, 2015 (revolutions 1554-1563; [24]). We present here the analysis of the available IBIS/ISGRI data [25], obtained during revolutions 1554-1558 (18-28 June, 2015; MJD 57191-51201), which cover the epoch of intense flaring activity and the beginning of the outburst decay (see Fig. 1a). These observations provide data sensitive enough to study in detail the properties of the Comptonizing medium.

\section{Data analysis}

The IBIS/ISGRI data reduction was performed using the Off-line Scientific Analysis software (OSA; [26]) v10.2, using the latest calibration files. The data were processed following standard IBIS/ISGRI reduction procedures. The spectral extraction was performed using good time interval files (GTIs) of variable duration, defined to provide source spectra of comparable S/N regardless of the source flux. We extracted 602 IBIS/ISGRI spectra, with exposure times in the range 64 to $176000 \mathrm{~s}$, and binned the IBIS/ISGRI response matrix in the energy range $20-500 \mathrm{keV}$ using 28 channels of variable logarithmic widths. To remove potential background contamination, and the contribution of additional spectral components above $200 \mathrm{keV}[12,13,27]$ we restricted the spectral fits to the 20-200 keV energy range. In our fits we have ignored the energy bin around $50 \mathrm{keV}$ and added 3 per cent systematic errors to the spectral bins. The IBIS/ISGRI X-ray spectra were fit using XSPEC v12.8.2, adopting the $\chi^{2}$ statistics. Errors provided below are quoted at the $1-\sigma$ confidence level $\left(\Delta \chi^{2}=1\right.$ for one parameter of interest).

\subsection{Spectral modelling}

We used Comptonization models to fit our data: NTHCOMP [28, 29] and comprs [30]. These models provide a description of the continuum produced by thermal Compton up-scattering of soft $\mathrm{X}$-ray photons. NTHCOMP is parameterized by a power law index $\Gamma$, and an electron temperature $T_{\mathrm{e}}$. The COMPPS parameters are the electron temperature $T_{\mathrm{e}}$ and optical depth $\tau$. In both models we fixed the seed photon temperature, $T_{\mathrm{bb}}$ to $0.1 \mathrm{keV}$, as it could not be constrained even by the Swift/XRT data (Motta et al. 2016, in preparation). In some cases, a high-energy cutoff was either 


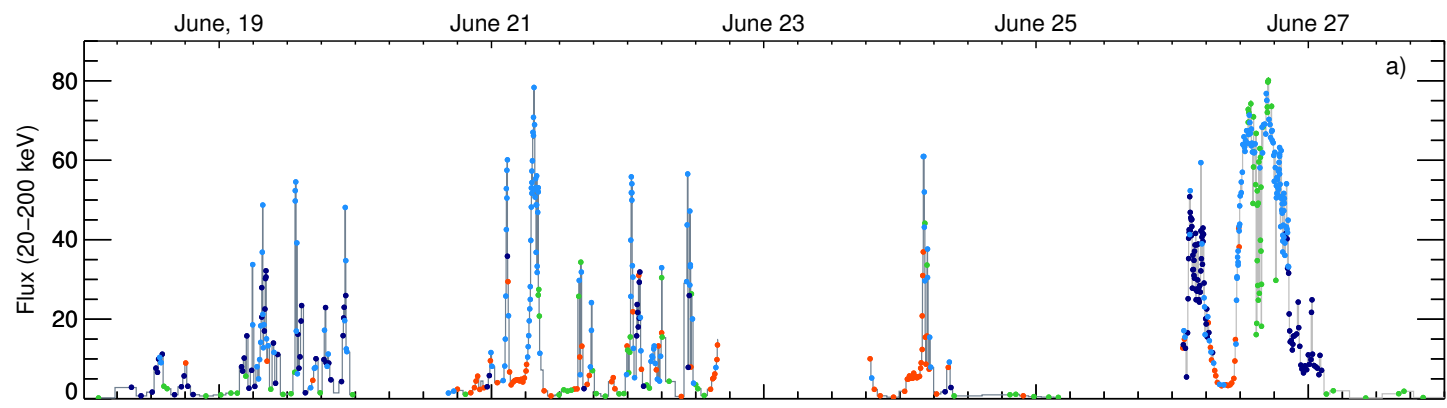

nthcomp

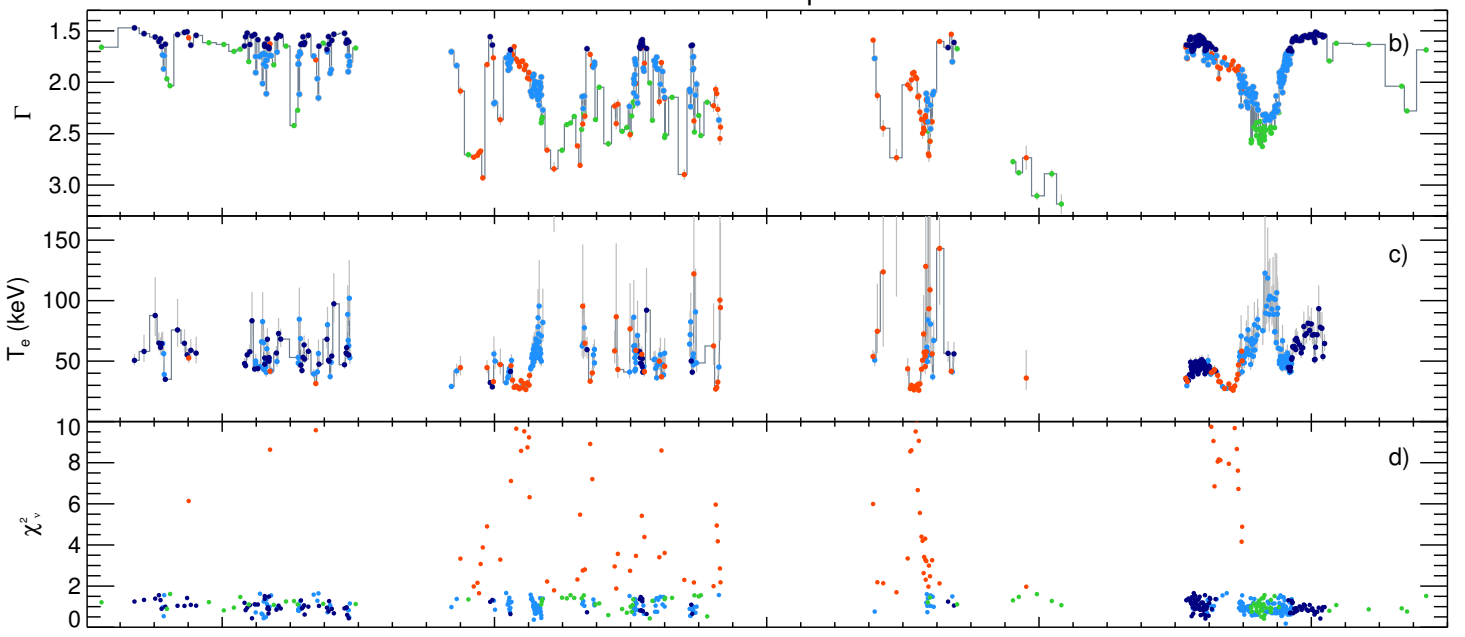

compPS

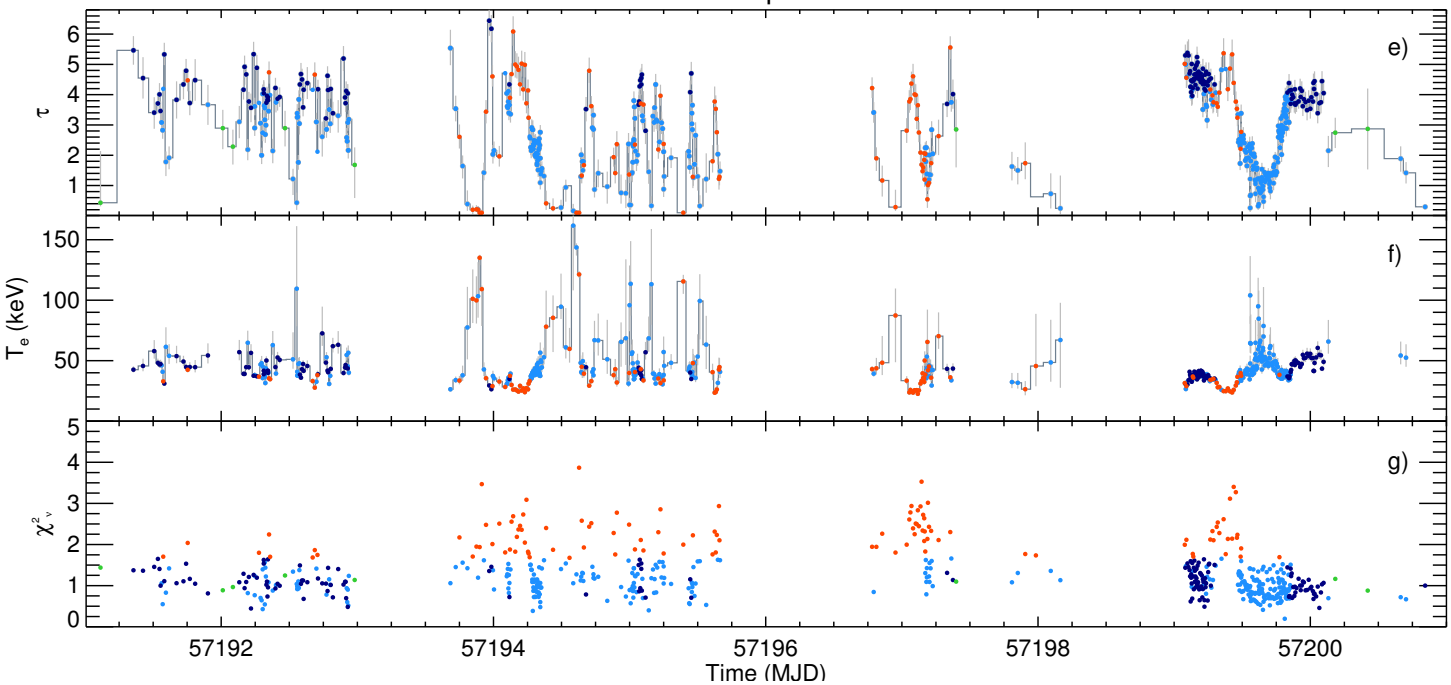

Figure 1: Time evolution of the flux and spectral parameters of V404 Cyg observed during the June 2015 flaring episodes. Panel a) source flux (20-200 keV) in units of $10^{-8} \mathrm{erg} \mathrm{cm}^{-2} \mathrm{~s}^{-1}$. Panel b), c), d) NTHCOMP fitting parameters (power-law index, $\Gamma$, electron temperature, $T_{\mathrm{e}}$ and $\chi_{v}^{2}$. Panel e), f), g) COMPPS fitting parameters (optical depth, $\tau$, electron temperature, $T_{\mathrm{e}}$ and $\chi_{v}^{2}$ ). Green, blue and red symbols are used to highlight the best-fitting model as follows: Comptonization models with constrained $T_{\mathrm{e}}$ (Fig. 2a) further divided into hard ( $\Gamma<1.7$; dark blue) and soft spectra ( $\Gamma \geq 1.7$; light blue). Green: Comptonization models with unconstrained $T_{\mathrm{e}}$ (Fig. 2b). Red: model $p$-test $<0.05$ fits. (Fig. 2c). 

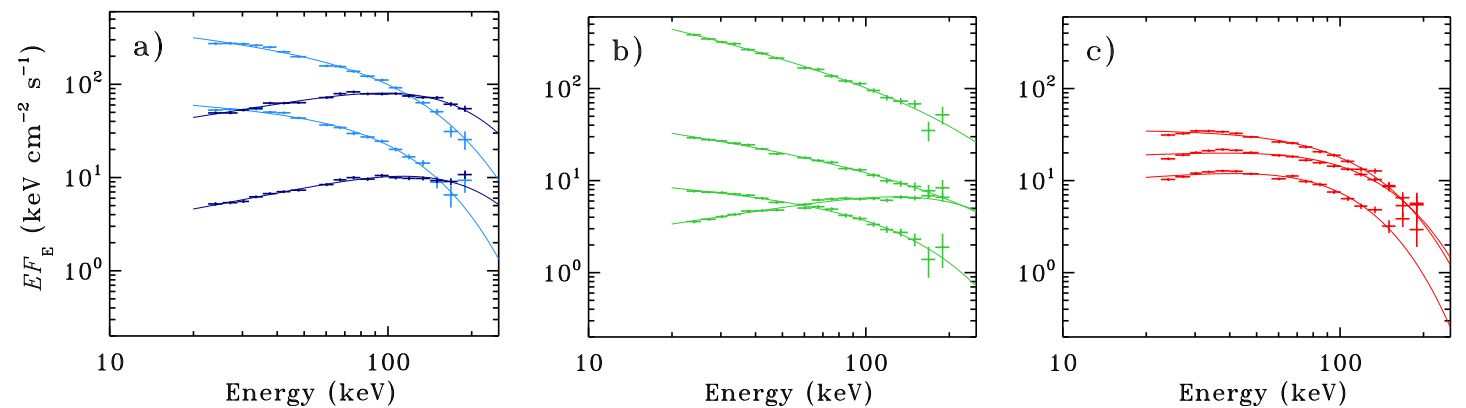

Figure 2: Example of the spectra analyzed in this work. Panel a) Comptonized spectra displaying a cutoff at high energies, classified in hard ( $\Gamma \leq 1.7$; dark blue) and soft ( $\Gamma>1.7$; light blue). Panel b) Comptonized spectra for which $T_{\mathrm{e}}$ cannot be constrained by our fits. Panel c) spectra for which Comptonization models were not statistically favored by our model selection criteria $(p$-test $<0.05)$.

weakly significant or not statistically required by the data. These spectra could still be fit using Comptonization models, but fixing $T_{\mathrm{e}}$ to an arbitrary high value $\left(T_{\mathrm{e}}=999 \mathrm{keV}\right)$. To account for both possibilities, we carried out two independent fitting runs per model: in the first run, we left $T_{\mathrm{e}}$, as a free parameter, while in the second run we fixed it to $T_{\mathrm{e}}=999 \mathrm{keV}$. Then, the Bayesian information criterion (BIC; [31]) was applied to select the best fit to the data. We computed the BIC using the approximation: $\mathrm{BIC}=\chi^{2}+k \ln (n)$, where $k$ is the number of parameters in the model, and $n$ is the number of channels in the spectral fits. We adopted $\Delta \mathrm{BIC}>6$ as the limit for model selection [32]. This approach was applied to the NTHCOMP and COMPPS fits. The results of this analysis are displayed in figures $1-3$, using the following color convention:

- Blue points highlight those fits where the $\Delta \mathrm{BIC}$ model selection favored a Comptonization model with a constrained $T_{\mathrm{e}}$ (see Fig. 2a), further divided in two groups: 'hard spectra' $(\Gamma<1.7$; dark blue) and 'soft spectra' ( $\Gamma \geq 1.7$; light blue).The latter classification is based on the $\Gamma$ values derived from the NTHCOMP fits, and then applied to the COMPPS fits.

- Green points correspond to those spectra where $T_{\mathrm{e}}$ could not be constrained by our data (i.e. $T_{\mathrm{e}}$ fixed at $999 \mathrm{keV}$; see Fig. 2b). .

- Additionally, for each fit we computed the corresponding $p$-value of the fit with respect to the data. We mark the spectra where $p<0.05$ with red symbols (see Fig. 2c). .

\section{Results}

We present in Fig. 1 the time evolution of the source flux computed in the 20-200 keV energy range, together with the evolution of the spectral parameters derived using NTHCOMP $\left(\Gamma, T_{\mathrm{e}}\right)$ and COMPPS $\left(\tau, T_{\mathrm{e}}\right)$ and the corresponding $\chi_{v}^{2}$ values. The relations between the spectral parameters derived from our fits are presented in Fig. 3. The light curve of V404 Cyg during the June 2015 outburst does not display the typical features of the standard BHB light curves (e. g. [33, 34]). Similarly, the soft X-ray spectra of V404 Cyg is remarkably different from the spectra of other BHBs, mostly due to the extreme V404 Cyg intrinsic absorption [35]. However, when we look at the source spectra in hard X-rays (above $20 \mathrm{keV}$ ) where the absorption is not as important, we find some similarities between V404 Cyg and other BHBs. 

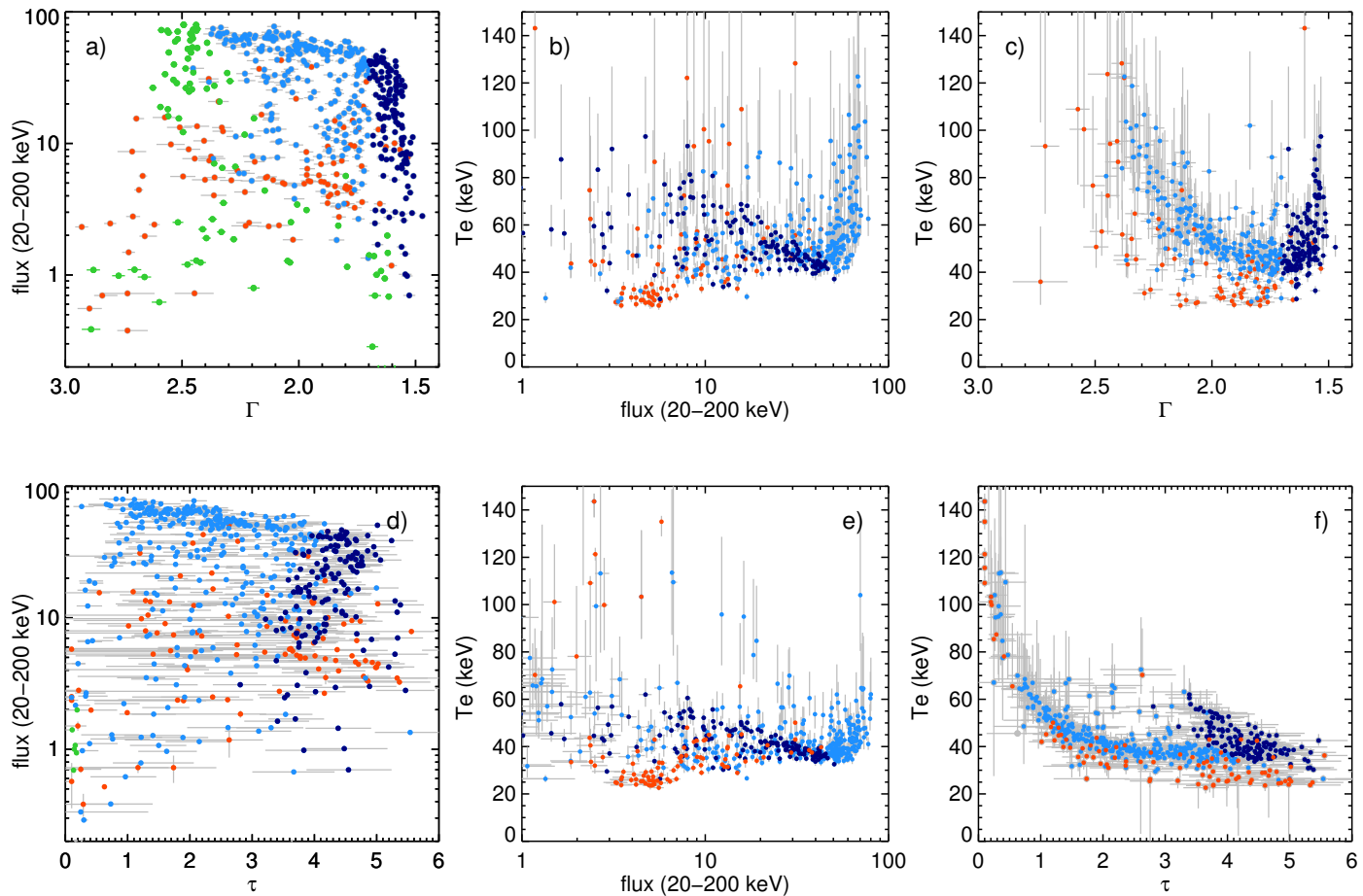

Figure 3: Relations between the parameters derived from our spectral fits, using the NTHCOMP (panels a-c) and the COMPPS models (panels d-e). Green, blue and red symbols are used to highlight the best-fitting model, according to our model selection criteria (Sect. 2.1). Panel a) $F_{\mathrm{X}}-\Gamma$ diagram. We find a hard branch (dark blue points) where $F_{\mathrm{X}}$ and $\Gamma$ are anti-correlated, similar to the hard state in the BHB HID. We also find a soft flaring branch (light blue points), where the source exhibits the highest fluxes in the outburst, but the $F_{\mathrm{X}}$ and $\Gamma$ anti-correlation persists. We identify the soft flaring branch with the BHB intermediate states. We also identify the softest spectra in our sample $(\Gamma \gtrsim 2.4)$ with unconstrained $T_{\mathrm{e}}$ with a tentative very high state (green points). Panel d) $F_{\mathrm{X}}-\tau$ diagram.Panel b, e) $F_{\mathrm{X}}-T_{\mathrm{e}}$ diagrams (NTHCOMP and COMPPS). Panel c) $\Gamma-T_{\mathrm{e}}$ diagram. Panel f) $\tau-T_{\mathrm{e}}$ diagram.

We identify a hard branch in the $\mathrm{F}_{X}-\Gamma$ diagram (Fig. 3a), occupied by the hardest spectra in our sample $(\Gamma \lesssim 1.7)$, which is reminiscent of the hard state in the HID [36, 37, 38, 39]. When the source occupies the hard branch, the spectrum gradually softens and $T_{\mathrm{e}}$ decreases as the flux increases (Fig. 3a, 3d, 3b, 3e). Similar anti-correlations are found in other BHBs in the hard state, supporting our identification of the hard branch with the hard state of prototypical BHBs such as e.g. GX 339-4 [40]. In the hard branch $\tau$ increases with increasing flux (Fig. 3d); $F_{\mathrm{X}}$ and $T_{\mathrm{e}}$ are anti-correlated (Fig. 3b, 3e) while $\Gamma$ and $T_{\mathrm{e}}$ are anti-correlated (Fig. 3f).

The softest spectra in our sample $(\Gamma>1.7)$, detected during the brightest X-ray flares, occupy a distinct region in the $F_{X}-\Gamma$ diagram (Fig. 3a) which we will call hereafter the soft flaring branch. In the soft flaring branch the spectrum still softens as the flux increases (Fig. 3a) but most of the parameter dependencies are reversed with respect to the hard branch: $\tau$ is seen to decrease with increasing flux (Fig. 3d); $F_{\mathrm{X}}$ and $T_{\mathrm{e}}$ are correlated (Fig. 3b, 3e) and the $\Gamma-T_{\mathrm{e}}$ dependency is also reversed. In the $\tau-T_{\mathrm{e}}$ diagram, we find a range of $\tau$ values $(\tau \approx[2.5-4])$ for which $T_{\mathrm{e}}$ displays a roughly constant value $(\sim 40 \mathrm{keV})$. Below $\tau \lesssim 2.5, T_{\mathrm{e}}$ and $\tau$ are anti-correlated (Fig. 3f). 
For spectra softer than $\Gamma \gtrsim 2.4$ the spectral fits using NTHCOMP do not provide constrained electron temperatures. These soft spectra are detected at the highest $\left(\gtrsim 20 \times 10^{-8} \mathrm{erg} \mathrm{cm}^{-2} \mathrm{~s}^{-1}\right)$ and lowest $\left(\lesssim 2 \times 10^{-8} \mathrm{erg} \mathrm{cm}^{-2} \mathrm{~s}^{-1}\right) F_{\mathrm{X}}$ values. When detected at the highest fluxes, they occupy regions in the $F_{\mathrm{X}}-\Gamma$ diagram (Fig. 3a) reminiscent of the HID very high state.

Finally, we also observe that the spectra detected at fluxes $\mathrm{F}_{X} \sim 5 \times 10^{-8} \mathrm{erg} \mathrm{cm}^{-2} \mathrm{~s}^{-1}$ during $\mathrm{X}$-ray plateaus (red points in Fig. 1) occupy distinct regions in all these diagrams, separate from the Comptonized branches, confirming our classification of these spectra in a separate category. We call these regions plateau branch(es).

\section{References}

[1] J. Khargharia, C. S Froning, C. S. and E. L. Robinson, 2010, ApJ 716, 1105.

[2] J. Casares, P. A, Charles and T. Naylor, 1992, Nature 355, 614.

[3] J. C. A. Miller-Jones, P. G. Jonker, G. Nelemans, S, Portegies Zwart, V. Dhawan, W, Brisken, E, Gallo, M. P. Rupen, M. P., 2009, ApJL 706, 230.

[4] G. A. Richter, 1989, Information Bulletin on variable Stars 3362.

[5] F. Makino, 1989, IAU Circular 4782.

[6] B. G. Marsden, 1989, IAU Circular 4783.

[7] S. D. Barthelmy and B. Sbarufatti, 2015, GRB Coordinates Network 17963.

[8] H. Negoro, et al, 2015, The Astronomer's Telegram 7646.

[9] G. Younes, 2015, GRB Coordinates Network 17932.

[10] G. R. Sivakoff, A. Bahramian, D. Altamirano, A. P. Beardmore, E. Kuulkers, S. Motta, 2015, The Astronomer's Telegram 7959.

[11] A. Loh, S. Corbel, G. Dubus, J. Rodriguez, I. Grenier, T. Hovatta, T. Pearson, A. Readhead, R. Fender, K. Mooley, 2016, MNRAS 462, 111.

[12] J. Rodriguez et al, 2015, $A \& A$ 581, 9.

[13] J. P. Roques, E. Jourdain, A. Bazzano, M. Fiocchi, L. Natalucci, Lorenzo; P. Ubertini, 2015, ApJL 813, 22.

[14] P. A. Jenke, C. A. Wilson-Hodge, J. Homan, P. Veres, M. S.Briggs, E. Burns, V. Connaughton, M. H. Finger, M. Hui, M., 2016, ApJ 826, 37.

[15] D. Walton et al, 2016, AAS/High Energy Astrophysics Division 15.

[16] P. Gandhi et al, 2016, MNRAS 459, 554.

[17] M. Kimura et al, 2016, Nature 529, 54.

[18] T. Muñoz-Darias, J. Casares, D. Mata SÃąnchez, R. P. Fender, M. Armas Padilla, M. Linares, G. Ponti, P. A. Charles, K. P. Mooley, J. Rodriguez, 2016, Nature 534, 75.

[19] S. S. Eikenberry, et al, 2016, AAS/High Energy Astrophysics Division 15.

[20] A. Tetarenko, 2015, The Astronomer's Telegram 7708.

[21] A, Segreto, 2015, The Astronomer's Telegram 7755. 
[22] C. Ferrigno, E. Bozzo, R. Boissay, E. Kuulkers, P. Kretschmar, 2015, The Astronomer's Telegram 7731.

[23] D. Walton, et al, 2015, The Astronomer's Telegram 7752.

[24] E. Kuulkers, 2015, The Astronomer's Telegram 7758.

[25] F. Lebrun, 2003, $A \& A$ 411, 141.

[26] T. J.-L. Courvoisier et al, 2003, $A \& A$ 411, 53.

[27] T. Siegert, R. Diehl, A. C. Vincent, F. Guglielmetti, M. Krause, C. Boehm, 2016, Nature 531, 341.

[28] A. A. Zdziarski, W. N. Johnson, \& P. Magdziarz, 1996, MNRAS 283, 193.

[29] P. T. Życki, C. Done \& C. and D. A.A. A. Smith, 1999, MNRAS 309, 561.

[30] J. Poutanen \& R. Svensson, 1996, ApJ 470, 249.

[31] G. Schwarz, 1978, Annals of statistics 6, 461.

[32] R. Kass, \& A. Raftery, 1995, Journal of the American Statistical Association 90, 773.

[33] W. Chen, C. R. Shrader \& M. Livio, 1997, ApJ 491, 312.

[34] R. A. Remillard \& J. E. and McClintock, 2006, ApJ 44, 49.

[35] S. E. Motta, J. J. E. Kajava, C. Sánchez-Fernández, M. Giustini, E. Kuulkers, 2017, MNRAS 468.

[36] J. Homan, et al, 2001, ApJSS 6, 461.

[37] T. Belloni, 2004, Nuclear Physics B Proceedings Supplements 132, 337.

[38] R. P. Fender, T. M. Belloni \& E. Gallo, 2004, MNRAS 335, 1105.

[39] R. J. H. Dunn, 2010, MNRAS 403, 61.

[40] T. M. Belloni \& S. E. Motta, 2016, Astrophysics of Black Holes: From Fundamental Aspects to Latest Developments 440, 61. 